\title{
Finalized design of LHAASO electromagnetic particle detector
}

\author{
Chao Hou * \\ The Institute of High Energy Physics, Chinese Academy of Sciences \\ E-mail: houchaodihep.ac.cn
}

Xiangdong Sheng, Jia Liu, Hongkui Lv, Jing Zhao, Xiaopeng Zhang

The Institute of High Energy Physics, Chinese Academy of Sciences

\section{Zhongquan Zhang, Chenguan Zhu, Wei Gao for LHAASO collaboration}

School of Physics, Shandong University, Jinan, China

\begin{abstract}
LHAASO will lay 5242 electromagnetic particle detectors (EDs) in an area of 1 square kilometer above sea level at $4410 \mathrm{~m}$. ED will be used to detect the arrival time and particle number density of secondary electromagnetic particles in the extensive air showers which are generated by the primary cosmic rays and gamma rays with an energy range from $10 \mathrm{TeV}$ to $100 \mathrm{PeV}$. This paper mainly introduces the finalized design of ED, including the design of structures, long-term stability and consistency, as well as the test results of EDs built in batches.
\end{abstract}

36th International Cosmic Ray Conference -ICRC2019-

July 24th - August 1st, 2019

Madison, WI, U.S.A.

${ }^{*}$ Supported by the National Natural Science Foundation of China (Grant Nos.11805209 and 11605208 ) 


\section{Introduction}

Large High Altitude Air Shower Observatory (LHAASO) [W] is being built at 4410m above sea level near the Daocheng, in Sichuan province, China. LHAASO will be completed by the end of 2020. LHAASO is a hybrid EAS (extended atmosphere shower) array[[2], which contains KM2A (1 square KiloMeter Array), WCDA (Water Cherenkov Detector Array) and WFCTA (Wide-Field Cherenkov Array). KM2A, consists of 5242 electromagnetic particle detectors (EDs) and 1171 muon detectors (MDs) in an area of 1.3 square kilometers fig.W, is dedicated in searching for galactic gamma ray sources above $30 \mathrm{TeV}$ in the northern sky and measuring primary cosmic rays in the energy range of $10 \mathrm{TeV}$ to $100 \mathrm{PeV}[3]$. ED is designed to detect the particle density and arrival

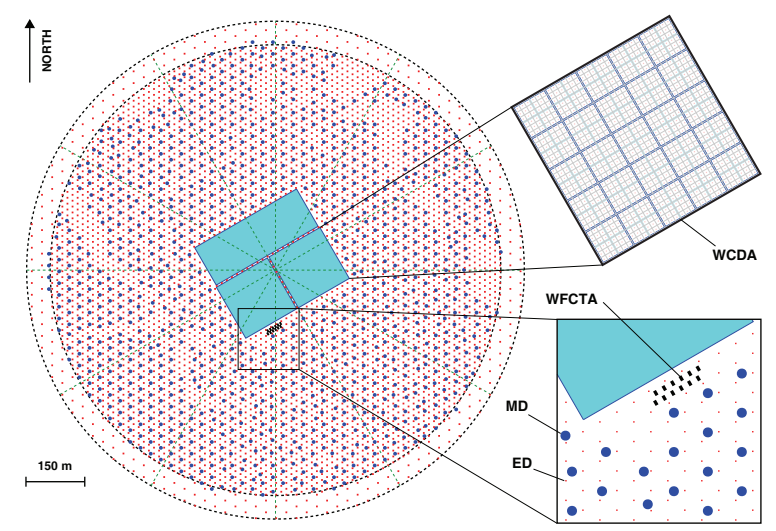

Figure 1: Sketch picture of LHAASO: The red points represent EDs and blue points are MDs, which constitute the KM2A.

times of secondary particles in EAS. The plastic scintillator is chosen as the sensitive detection medium because of its high detection efficiency to charged particles, quick response time and well stability. The performance requirements of unit ED are[B]]:

1. The sensitive detection of geometric area for a unit ED is $1 \mathrm{~m}^{2}$;

2. Measuring secondary electromagnetic particles, including $\mu^{ \pm}, \mathrm{e}^{ \pm}, \gamma$;

3. The dynamic range of ED should cover 1-10000 particles $/ \mathrm{m}^{2}$. Measurement accuracy: $25 \%$ @ single particle, $5 \%$ @ 10000 particles.

4. The time resolution should be better than $2 \mathrm{~ns}$ to reach a good angular resolution and high sensitivity for the whole array.

5. The total detection efficiency for charged particles should be better than $95 \%$.

6. The count rate should be less than $2 \mathrm{kHz}$ (at working gain and given threshold).

In addition, all EDs should have uniformity within $10 \%$ for the whole array to obtain an unbiased measurement of EAS. 


\section{Finalized design}

In the ED finalized design, a combination of detector simulation and prototype experiment is used. The factors that influence the long-term stability of the ED, cost-effective and easy to mass production are taken into consideration.

\subsection{Construction}

As a result of ED optimized design, "Plastic Scintillator tile + Wavelength Shifting Fiber" constitutes a detection sensitive unit. The size of scintillator is $100 \mathrm{~cm} \times 25 \mathrm{~cm}$ (length $\times$ width). Grooves are machined on the surface of the scintillator along the width direction for the placement of fibers. A fiber ( $2.7 \mathrm{~m}$ in length) is passed through the scintillator and reentered in an alternate groove. Each scintillator is wrapped with a layer of Tyvek to increase photon collection efficiency and a layer of black cloth to avoid external and inter-cell optical crosstalk[四], as shown in fig.[. Four detection sensitive units are assembled symmetrically to cover the $1 \mathrm{~m}^{2}$ detection area. All fibers end faces are bunched together for polishing the surface, and then coupled to the central location of photoelectron multiplier tube (PMT) cathode directly fig.9. When the charged particles pass through the ED scintillator, the energy deposited by ionization and alike is converted to fluorescent photons. The photons are collected by the fiber, and the second light is emitted and propagated to the cathode surface of the photomultiplier tube. Here, through the process of photoelectric conversion, multiplication, etc., the output signal is finally formed through the dynode and the anode, and sent to the subsequent electronic system. Digitized charge and time information is collected and recorded[䧃].

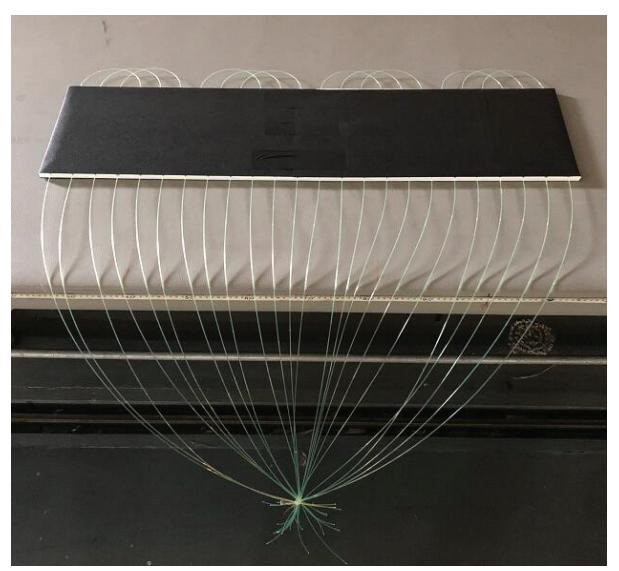

Figure 2: one of ED detection sensitive unit

The thickness of scintillator and the density of fiber will sensitively affect the light output of the detector which is equivalent to the number of photoelectrons (npe) emitted by the photocathode and the charge output from PMT. According to the performance requirements of ED, the specification of design test index could be concluded as: the peak of vertical single muon charge spectrum should be around 20pe (photon electron), the resolution should less than $25 \%$. The time resolution should be better than 2 ns. The uniformity of npe between EDs should within $10 \%$. The total detection efficiency for charged particles should be better than $95 \%$. 
Based on the Geant 4.9.3 version, a simulation program for the ED detection sensitive unit for simulation of detector performance is developed. Detector simulation mainly includes the events generation, the interaction between particles and detector materials, propagation and the process of digitization. The program sets the material properties, the geometry and the properties of the interface between the materials. The photon track, energy, momentum, time and other information of each process in the detection sensitive unit are recorded for further obtaining the signal of the detector response. Fig. [3] shows the simulation of photons propagating in ED after a muon passes through an ED detection sensitive unit.

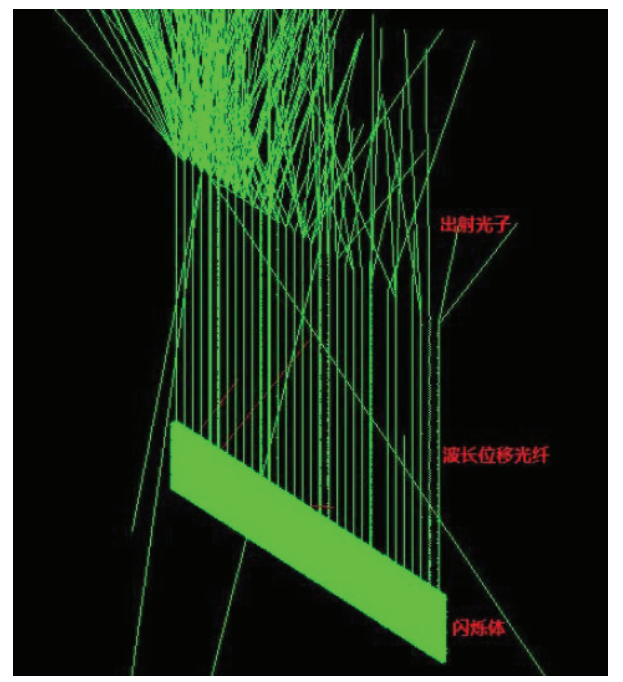

Figure 3: Simulation of photons propagating in ED after a muon passes through an ED detection sensitive unit

The laboratory uses the telescope method to test the photoelectron number and time resolution of the sensitive unit. The probe size is $25 \mathrm{~cm} \times 25 \mathrm{~cm}$. The tested detection sensitive unit is placed in the middle of the probes with a spacing of $10 \mathrm{~cm}$. During the test, the PMT used is EF00014 of CR285-01 with a gain of $4 \times 10^{5}$ and a working high voltage of $944 \mathrm{~V}$. The fiber uses 32 single cladding BCF-92SC. The fiber end face is directly coupled to the PMT photocathode surface. The single particle spectrum obtained by the test is fitted by landau function, and the fitted MPV (Most Probable Value) is the npe of the sensitive unit. The npe for $1.0 \mathrm{~cm}$ thick BC408 scintillator is $26.1 \pm 0.5$, shown in fig.t. The error consists of a 0.1 pe statistical error and a $2 \%$ systematic error. The output number of photons is obtained by simulation, the npe can be obtained by the conversion of quantum efficiency $(19.8 \%)$ of PMT. A single particle spectrum was obtained by simulating a $1.0 \mathrm{~cm}$ thick BC408 scintillator. The npe is 25.8 as shown in fig. 1 . It can be seen that the simulated and experimental npe are very close, and proves that the simulation program is more accurate than experiment for the single particle resolution.

Using the simulation program, the correspondence between Npe and the thickness of the scintillator in fig. 6 and the number of fibers shown in table.⿴囗十 is obtained [ $[\mathbf{\square}]$. Using these relationships, we derive a combination of the number of different thickness scintillators and fibers that can satisfy npe approximately equal to 20 . Through experiments, we can get the number of fibers can not be less than 20 to ensure that the time resolution is less than $2 \mathrm{~ns}$. At the same time, in order to ensure 

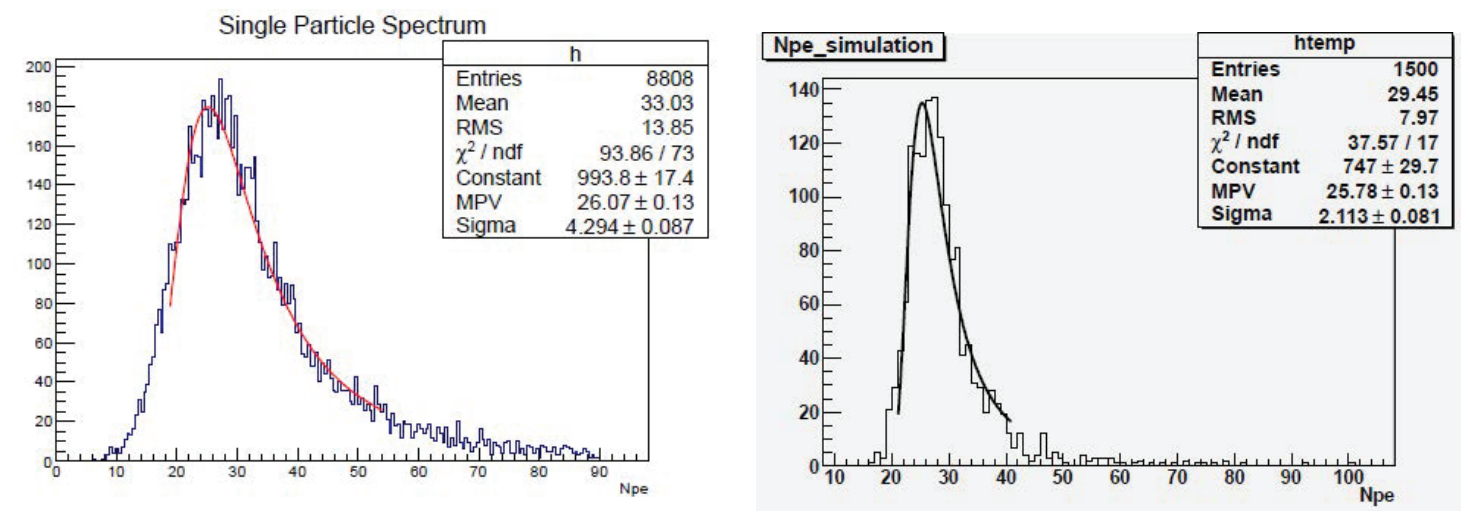

Figure 4: Experiment test result of single muon spec- Figure 5: Simulation result of single muon spectrum trum

the strength of the scintillator, the thickness cannot be less than $1 \mathrm{~cm}$. Finally, we get a sensitive unit with a number of fiber of 24 and a fiber length of 2.7 meters. The thickness of different candidate types of scintillator are calculated. For the type of BC408 (ED used), the thickness is $1 \mathrm{~cm}$. Such combinations have been experimentally tested to meet design specifications[प].
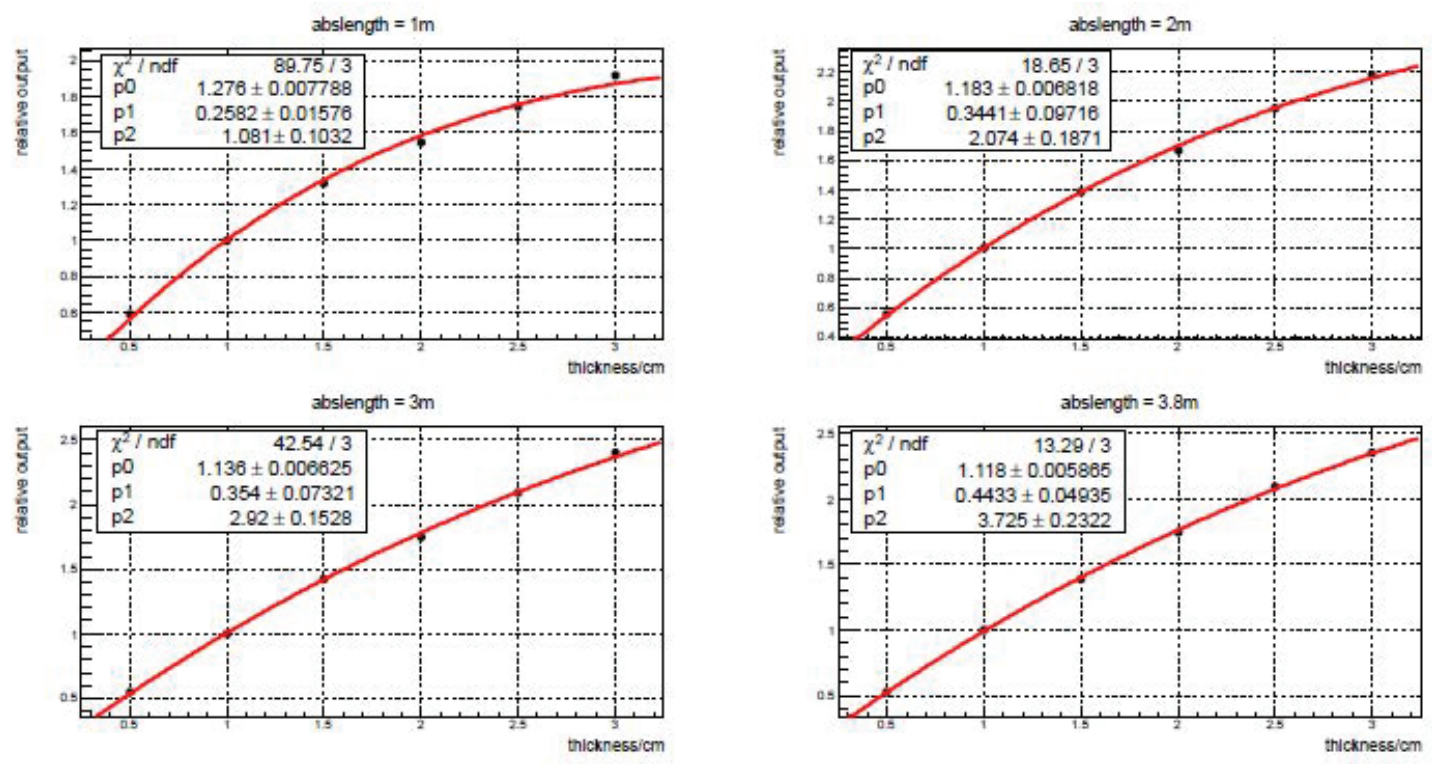

Figure 6: Simulation results of npe and plastic scintillator thickness. The four graphs show the relationship between Npe and the thickness of the scintillator when the scintillator attenuation length is $1 \mathrm{~m}, 2 \mathrm{~m}, 3 \mathrm{~m}$ and $3.8 \mathrm{~m}$. Use the function $\mathrm{f}(\mathrm{x})=\mathrm{p} 0 \cdot \mathrm{x} \cdot \exp (-\mathrm{p} 1 \cdot \mathrm{x} / \mathrm{p} 2)$ to fit the results.

\subsection{Design for ED Long term stability}

LHAASO will run for more than 20 years, requiring 10 years of performance reduction of no more than $20 \%$, and the materials and electronics used in ED will change over time, so many considerations and works have been done on the detector design and fabrication process. 
Table 1: Relative npe of the fibers for the different quantities (columns) and thicknesses of scintillators (rows).

\begin{tabular}{|c|c|c|c|}
\hline & $2 \mathrm{~cm}$ & $1.5 \mathrm{~cm}$ & $1.0 \mathrm{~cm}$ \\
\hline 32 & 1.40 & 1.44 & 1.45 \\
28 & 1.32 & 1.35 & 1.37 \\
24 & 1.21 & 1.25 & 1.27 \\
20 & 1.12 & 1.14 & 1.14 \\
16 & 1.00 & 1.00 & 1.00 \\
12 & 0.86 & 0.83 & 0.84 \\
8 & 0.69 & 0.67 & 0.66 \\
\hline
\end{tabular}

The aging and stability of the core part scintillator and fiber are studied. The laboratory has setup a high-temperature accelerated aging experiment, and estimated the service life of each candidate scintillators and fibers are more than 10 years with a performance reduction of $20 \%$ in the environment of Daocheng site (annual average temperature is $1^{\circ} \mathrm{C}$ ). In terms of detector design, the coupling between fiber and scintillator, fiber and photomultiplier tube adopts the most stable air coupling method. At the same time, the PMT is subjected to long-term measurement of high-frequency pulse signals. When the accumulated charge is $20 \mathrm{C}$, the gain does not change significantly, meeting the service life requirement. Also, the development of the power system focused on the design of low air pressure, moisture resistance, long-term reliability, etc. The temperature effect of the charge of electronics and PMT is studied. As a result, $0.06 \% /{ }^{\circ} \mathrm{C}$ is for ED electronic system, and $0.2 \% /{ }^{\circ} \mathrm{C}$ is for PMT.

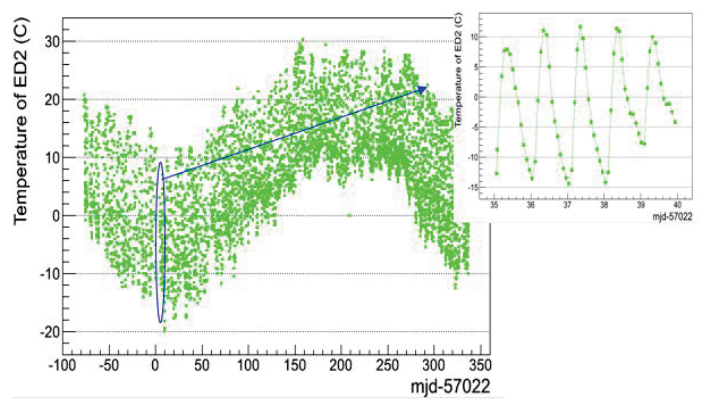

Figure 7: Temperature monitoring result for ED2

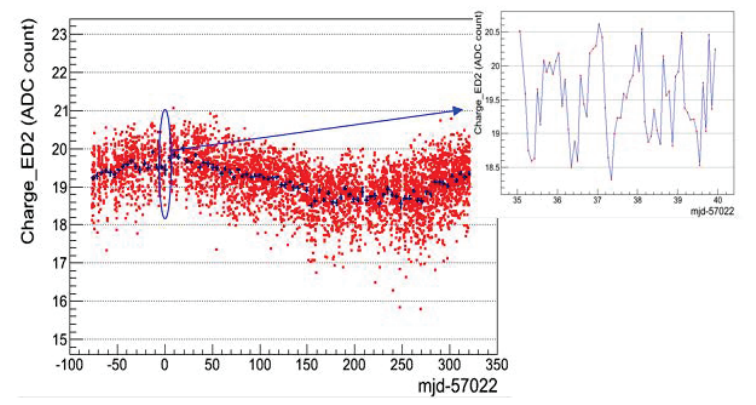

Figure 8: MPV of charge distribution monitoring for ED2

The stereotype of ED innner structure can be seen in fig.Q. According to the finalized design, we had built a prototype array in Yangbajing, Tibet. One year's test results shows that the amount of charge drops less than $2 \%$ as shown in fig. 8 .

\section{The performance test results of EDs built in batches}

The performances are evaluated with the single muon particles selected by a telescope system, which is established and calibrated in Shandong University. The system could measure the performance of ED in different positions [焑]. 


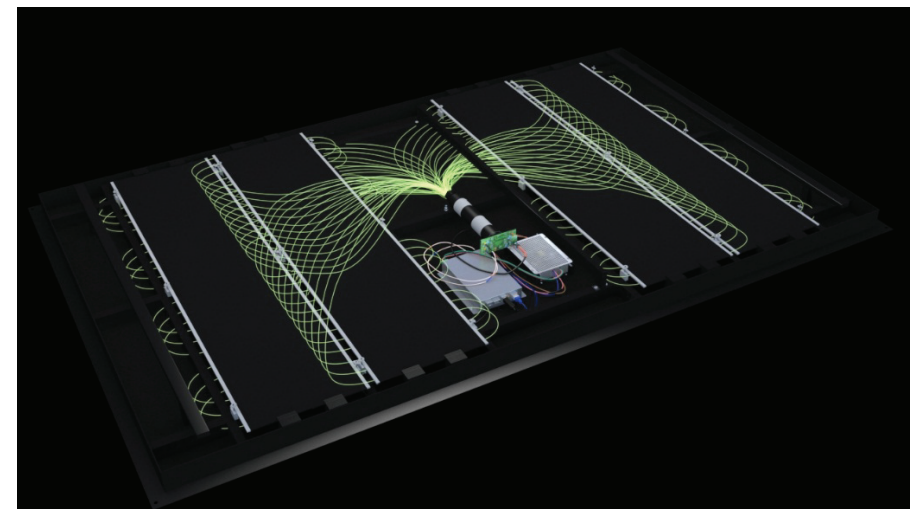

Figure 9: Stereotype of ED Inner structure

\section{Single particle energy spectrum test and scanning}

Single particle Charge spectrum is acquired and fitted with the convolution of Landau with Gaussian function, as shown in fig. 10 . The peak is used to represent charge output of ED, and Npe is calculated[1]. The charge scanning result is shown in fig. 1 .

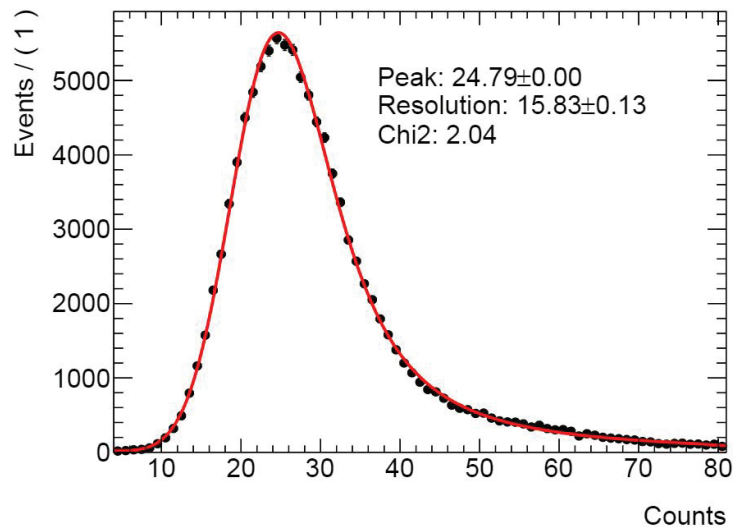

Figure 10: Single particle charge spectrum

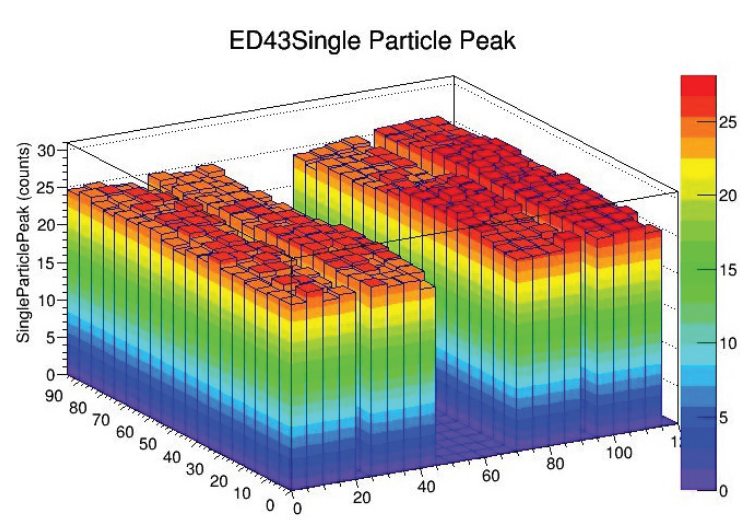

Figure 11: Scanning result of Single particle peak for a ED

\section{Time resolution scanning}

In the ED time resolution measurement, the hit time of the ED measurement and the hit time of the system measurement are compared, and the distribution of the difference will include the system time resolution and the ED time resolution. The ED time resolution can be obtained by subtracting the contribution of the system[[]. A ED time resolution scan results are shown in Fig.[12.

\section{Count rate}

Count rate refers to the signal rate that exceeds the preset threshold of electronics, which is mainly contributed by the MIP signal, dark noise of PMT and background radiation[5]. The count rate of all detectors are less than $2 \mathrm{kHz}$ at LHAASO site.

The detection efficiency sanning result is shown in fig. [33. The performance test results of batches of ED are list in table $\square$. All performance meets the requirements of the indicator. 


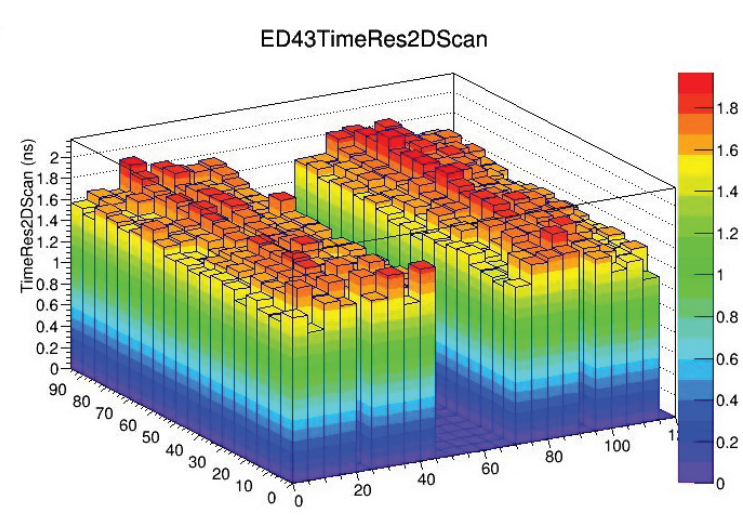

Figure 12: Time resolution

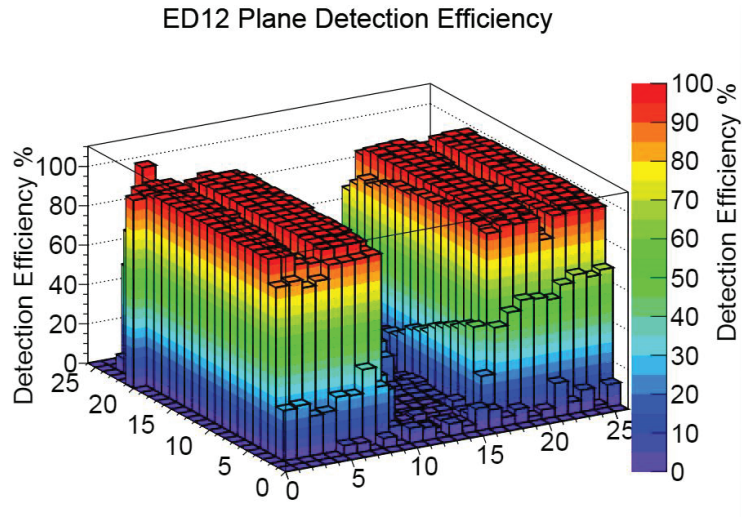

Figure 13: Detection efficiency

Table 2: Performance test results of the unit ED. npe (by mean; no Lead plate).Single muon spectral resolution (fitted by landau function)

\begin{tabular}{|l|l|l|}
\hline Detector performance & Requirement & Batch test results \\
\hline mean npe & $>20$ & $21.0 \pm 0.9$ \\
Single muon spectral resolution & $<25 \%$ & $<20 \%$ \\
Time resolution & $<2 \mathrm{~ns}$ & $1.62 \pm 0.05 \mathrm{~ns}$ \\
Detection efficiency & $>95 \%$ & $\sim 98 \%$ \\
Npe uniformity & $<10 \%$ & $8.6 \%$ \\
\hline
\end{tabular}

\section{Conclusion}

Through the combination of simulation and experiment, considering the ED stability factor, cost-effective and easy to mass production, the ED design is completed. The performance test of the batches ED meets the design specification requirements.

\section{References}

[1] Z. Cao, For the LHAASO Collaboration, Status of LHAASO updates from ARGO-YBJ, Nucl.Instrum. Methods Phys. Res. A 742 (2014) 95-98.

[2] H. H. He, For the LHAASO Collaboration, Design of the LHAASO detectors, Radiation DetectionTechnology and Methods (2018) 2.

[3] Feasibility study report of LHAASO, IHEP-LS-PM-TR-16-001-R3, 2017.

[4] Z. Q. Zhang, C. Hou et al., Study on the performance of electromagnetic particle detectors of LHAASO-KM2A, NIM A, vol. 845, p. 429-433, 2017.

[5] ED overall identification acceptance report, 2018.

[6] T. Y. Xu, Y. Y. Du et al., Test of the LHAASO electron detector prototype using cosmic rays, Chinese Physics C, vol. 38, No. 11 (2014) 116004.

[7] Zhongquan Zhang, Study and optimization on electromagnetic particle detectors in LHAASO-KM2A. 